\title{
DOS CUENCOS AQUEMÉNIDAS DE BRONCE EN COLECCIONES MADRILEÑAS
}

\author{
TWO ACHAEMENID BRONZE BOWLS IN MADRID COLLECTIONS
}

\author{
MARTÍN ALMAGRO-GORBEA \\ Real Academia de la Historia \\ JOAQUÍN LÓPEZ PASCUAL \\ Colegio Universitario de estudios Financieros
}

\section{CUENCO DE BRONCE DE UNA COLECCIÓN MADRILEÑA}

Una colección madrileña conserva desde hace años un cuenco de bronce procedente del mercado internacional de antigüedades, que, por sus características, merece la pena que sea dado a conocer, a pesar de que, por desgracia, se desconoce toda noticia sobre su procedencia y su contexto arqueológico originario (Fig. 1,a y b). La pieza llamó nuestra atención al estudiar el cuenco fenicio procedente de Nubia adquirido por la Real Academia de la Historia (Almagro-Gorbea, 2003, 7-32), pues ofrecía algún detalle común de interés, como las lengüetas del umbo, por lo que parece oportuno publicarlo junto con otro de características relacionables conservado en la Real Academia de la Historia (Fig. 10).

La pieza de la colección madrileña es un cuenco de bronce que ofrece una forma característica, constituida por un cuerpo inferior poco profundo y un amplio borde exvasado. Está realizado posiblemente por medio de batido a torno, aunque las numerosas concreciones que cubren sus paredes impiden precisar con seguridad este detalle, pues, además, la estructura maciza de la base tampoco permite excluir que la pieza se hubiera fundido. El cuerpo inferior es de forma muy aplanada, sin los gallones que ofrecen otras piezas parecidas, y ofrece una marcada curvatura reentrante en su parte superior, de la que sale el borde, casi vertical pero exvasado y ligeramente inclinado hacia fuera en su parte superior, con el borde de perfil redondeado.

El elemento más singular de esta copa puede considerarse el característico ónfalo central, que parece rodeado de ovas (Fig. 2), lo que permite considerarla con seguridad como una phiale (Luschey, 1939). El ómfalos o umbo central es de pequeño tamaño, pues apenas sobresale ligeramente sobre el fondo del vaso 4 $\mathrm{mm}$. Su contorno externo queda resaltado por un doble círculo concéntrico, de $19 \mathrm{~mm}$ y $26 \mathrm{~mm}$ de diámetro, que, probablemente, más que hechos a torno, parecen haberse trazado a mano alzada directamente sobre el molde de la pieza con un instrumento de punta relativamente roma. Sobre el círculo exterior del umbo y rodeándolo se han trazado una serie de 22 ovas dispuestas a modo de pétalos formando un círculo concéntrico, lo que da al umbo un cierto aspecto de una gran roseta central, con la base en el círculo exterior que lo delimita, y que resaltaría en el fondo del líquido bebido o libado en este vaso.

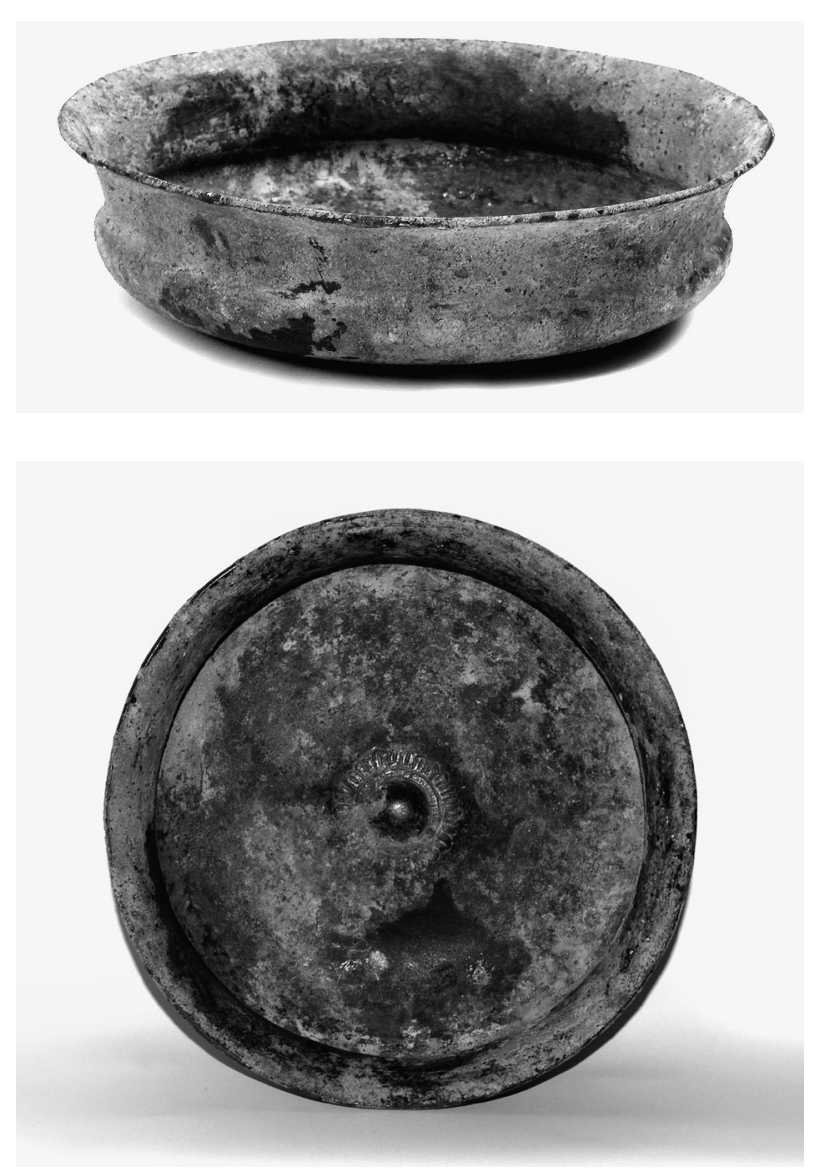

Figura 1 a-b: Phiale de bronce del Luristán de una colección madrileña. 


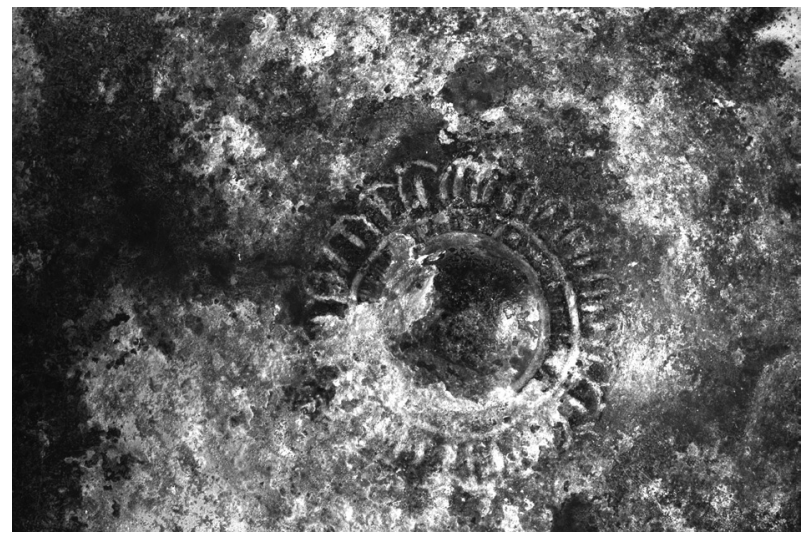

Figura 2: Detalle del umbo decorado con ovas.

Las ovas están trazadas de forma bastante esquemática y a mano alzada con un instrumento de punta roma como el utilizado para los círculos que rodean el umbo. Se han trazado haciendo un semicírculo con la base apoyada hacia el interior del umbo, en cuyo centro se ha dispuesto un trazo perpendicular que da a este motivo la forma característica de ova. En su trazado, en ocasiones los trazos de una ova se apoyan sobre la de al lado y permiten comprobar que aparecen trazadas de derecha a izquierda, lo que indica que si se hubieran trazado sobre un molde, se habrían realizado originariamente de izquierda a derecha. El diámetro máximo de esta banda circular de ovas es de $36 \mathrm{~mm}$., tras las cuales la pared del vaso baja para formar la base, por lo que las ovas y el umbo quedan ligeramente resaltados, tal como se observa con más claridad por el exterior.

El estado de conservación del vaso puede considerarse bueno en general, sin roturas ni abolladuras, e incluso ofrece algunas partes con el brillo especular originario por haber sido pulido, aunque la mayor parte de la superficie aparece cubierta de una ligera capa de concreciones estables de cuprita y óxidos de cobre y calcáreas.

Dimensiones: Diámetro máximo: $162 \mathrm{~mm}$. Altura máxima: $42 \mathrm{~mm}$. Peso: 260.20 gr. Capacidad de la panza: $295 \mathrm{~cm}^{3}$; Capacidad hasta el borde: $680 \mathrm{~cm}^{3}$.

Análisis metalográfico: $\mathrm{Cu} 85,1 \% ; \mathrm{Sn} 14.0 ; \mathrm{Pb}$ $0.73 \%$; Fe 0.12\%; Ni nd; Zn nd; As nd; Ag 0.027\%; Sb nd; Bi nd ${ }^{1}$.

\footnotetext{
1. Análisis efectuado con el espectómetro de fluorescencia de rayos X (energías dispersivas) Metorex XMET920 del Laboratorio del Museo Arqueológico Nacional por el Dr. Salvador Rovira, a quien agradecemos su colaboración siempre eficaz y amistosa. Según su informe metalográfico (14.5.2009), este cuenco del Luristán está hecho con un bronce binario $\mathrm{Cu}-\mathrm{Sn}$ apto para ser laminado y trabajado a martillo para darle forma, que responde a una tecnología de tradición antigua, que se remonta a la Edad del Bronce.
}

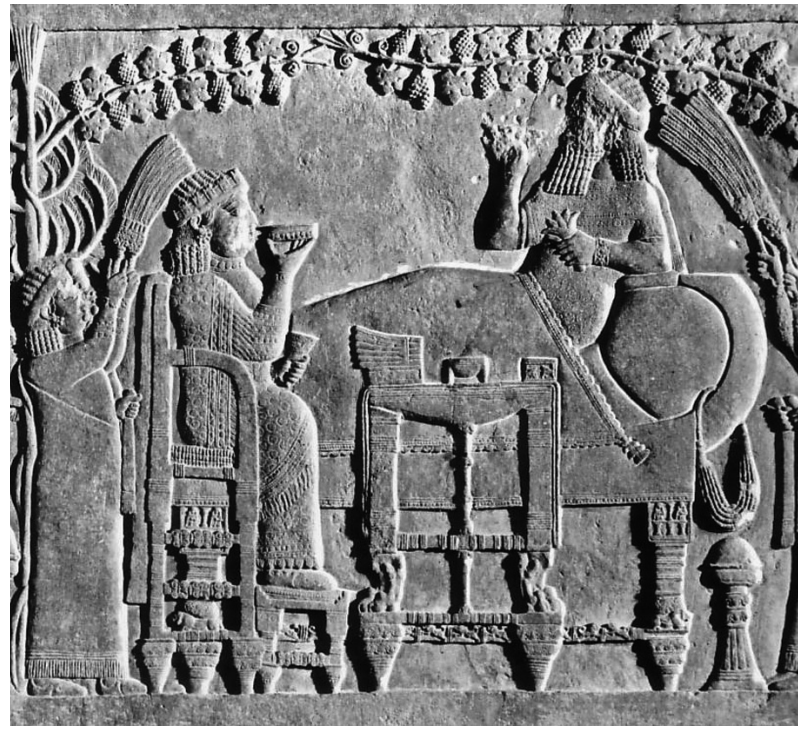

Figura 3: Relieve asirio de Nínive con el banquete de Asurbanipal III.

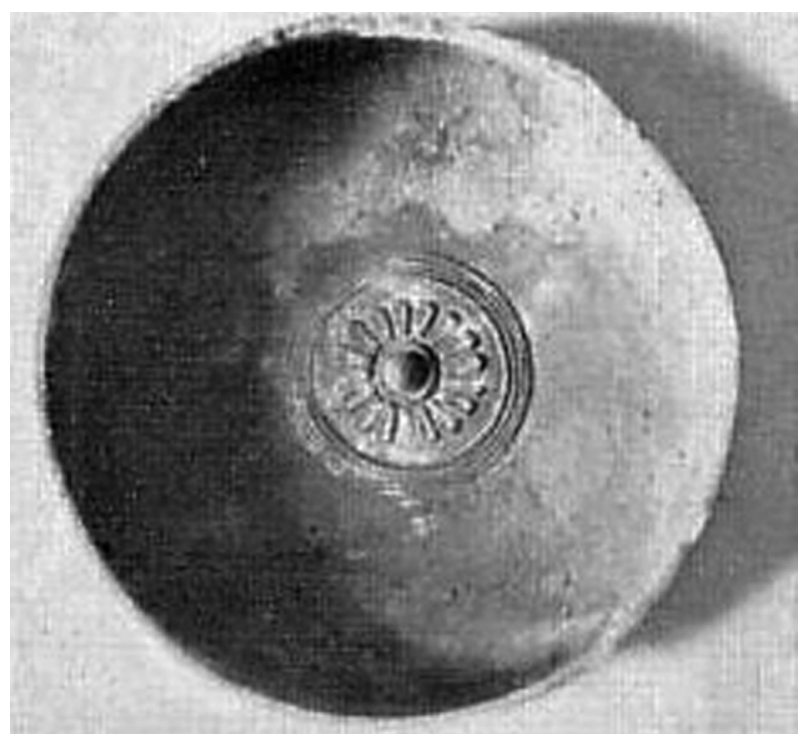

Figura 4: Cuenco de Asur conservado en el Museo de Berlín (según Luschey, 1939).

\section{PARALELOS}

Este tipo de cuenco se inscribe en una amplia serie de vasos muy característicos del Oriente. Su forma parece originaria de la región sirio-asiria, donde pueden encontrarse precedentes cerámicos para esta forma desde el Calcolítico hasta la Edad del Hierro (Ayoub, 1982, 116 s., tipo 69; Nigro, 2002, 118, nº 35-43). En todo caso, la popularidad de su uso explica que incluso aparezcan representados en relieves (Fig. 3), como el del banquete del Triunfo de Asurbanipal III (669-628 a.C.), en alguno de Nínive (Schneider-Herrmann, fig. 7 y 8) o en los relieves de Darío (521-486 a.C.), en Persépolis (Figs. 8 y 9; vid. infra). 

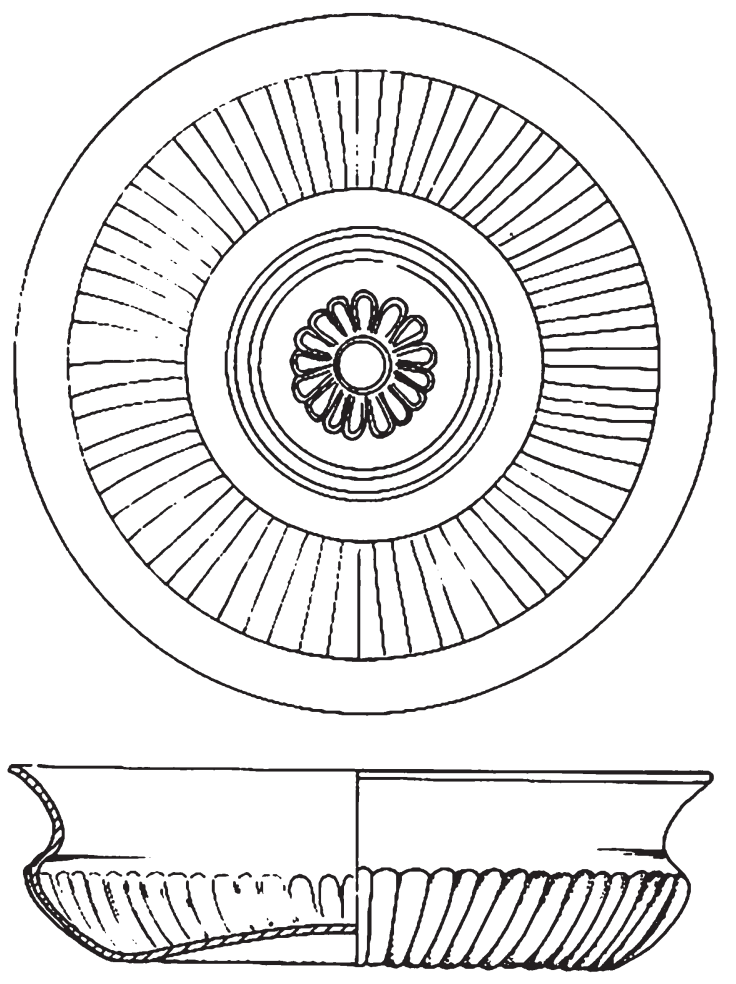

Figura 5: Cuenco del Luristán de la antigua colección Godard (según De Waele, 1982).

La popularidad de esta forma explica que este tipo de vaso fuera ampliamente imitado en el Luristán, uno de los principales centros de toréutica del Oriente, cuyos productos han pasado de forma habitual al mercado de antigüedades para surtir museos y colecciones, si bien la procedencia precisa de dichos bronces viene siendo discutida (Muscarella, 1988, 33 s.), pues no existe una monografía actualizada de estas phiales desde el estudio de H. Luschey (1939).

El umbo rodeado de ovas en un cuenco de forma sencilla de simple casquete esférico aparece en piezas asirias de Asur y en otras de borde exvasado de Nínive (Fig. 4), lo que pudiera indicar un origen asirio de este detalle (Luschey, 1939, fig. 1; Mallowan, 1966, 116, fig. 59), que parece ser la simplificación de decoraciones más complejas (Godard, 1931, 92, lám. 63, n 226), aunque piezas semejantes han aparecido en el Norte de Siria, fechadas en el último cuarto del siglo VIII a.C. (von Luschan, 1943, 118, fig. 165, lám. 56i). Sin embargo, la pieza de la colección madrileña ofrece el borde exvasado y el cuerpo liso, con las características ovas en torno al umbo central de la base que caracterizan muchos de estos cuencos considerados procedentes del Luristán.

Las excavaciones en Luristán han proporcionado ejemplares que confirman la procedencia de los existentes en colecciones y museos, aunque generalmente con el borde exvasado asociado a un cuerpo agallonado y al umbo central con o sin ovas, todo
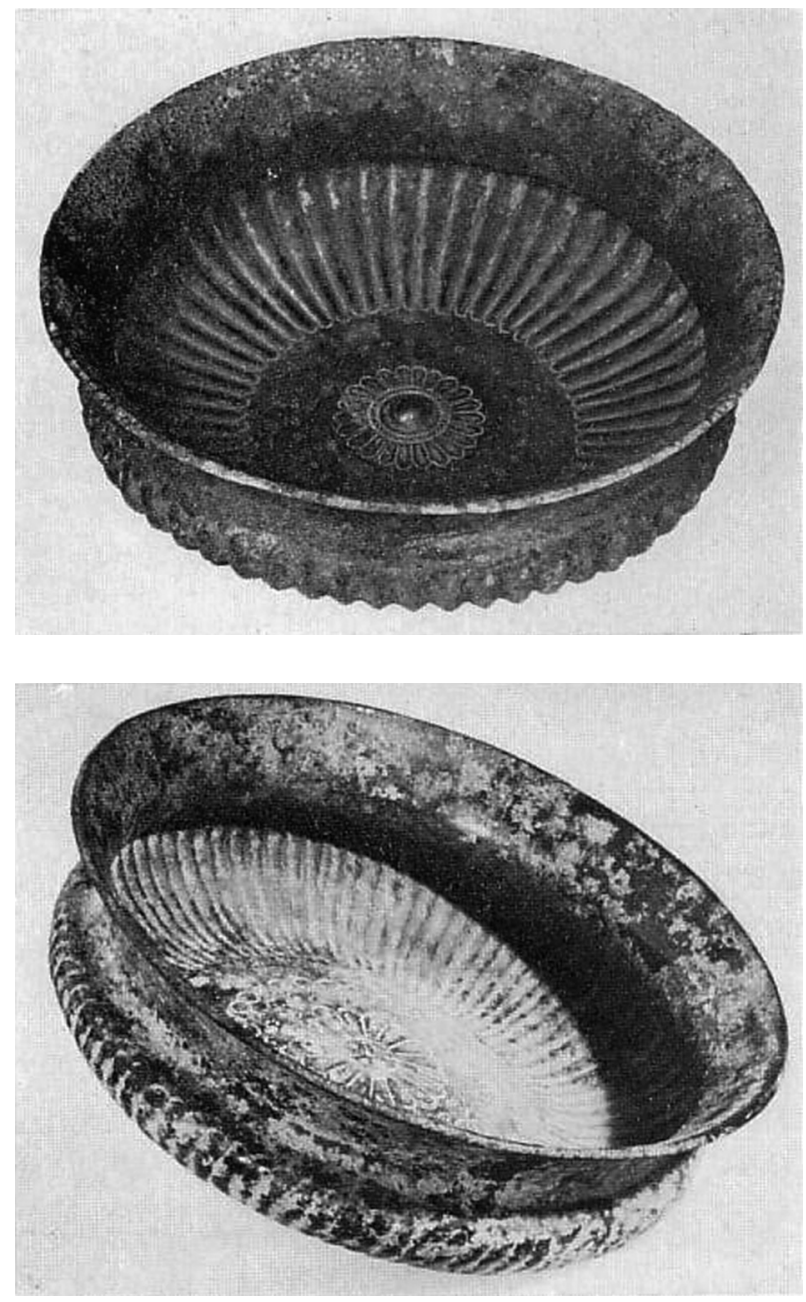

Figura 6: Cuenco del Luristán de la colección Adam (según Moorey, 1974).

ello más o menos marcado. Un cuenco sencillo con umbo rodeado de ovas procede de War Kabud y otro, algo más complejo, de Chamahzi Mumah (Vanden Berghe, 1968, lám. 32; Haerenick y Overlaet, 1998, 25 s., fig. 11 y 18, lám. 56,a). Como otros ejemplos sin procedencia precisa pueden considerarse un vaso de la antigua colección Godard, fechado en los siglos VIII-VII a.C. por De Waele $(1982,220$, no 372, fig. 193 y 199) y otros de Asur (Luschey, 1939, fig. 16,ac y 29 ,a-b) y Tell Halaf (Luschey, fig. 24; Calmeyer, $\left.1964, n^{\circ} 109\right)$, que ofrece el mismo pequeño umbo característico y dimensiones muy parecidas, pues mide 136 por $38 \mathrm{~mm}$ (Fig. 5). Otro ejemplar de esa misma colección, ligeramente mayor y con gallones más suaves pero de la misma cronología, de 148 por $41 \mathrm{~mm}$., ofrece lengüetas en torno a un círculo central (De Waele, 1982, 219, n 370, fig. 191 y 209), como otros paralelos semejantes de Asur (Luschey, 1939, fig. 1, 2), Djub-i Gauhar (Vanden Berghe, 1979, 146, fig. 5, no 15), Nimrud (Mallowan, 1966, 116, lám. 59) y Sendjirli (Andrae, 1943, lám. 56,e; Luschey, 1939, fig. 3), etc. 
También con ovas aparece decorada la base de un cuenco de cuerpo agallonado de la colección Bröckelschen, considerado de época neoasiria (Calmeyer, 1964, 53, no 110), las de otros dos cuencos agallonados (Fig. 6) de la Colección Adam (Moorey, 1974, nº 129 y 130) y las de otros ejemplares de la colección David-Weill (Amiet, 1976, 96-97) y del Museo de Leiden (Schneider-Herrmann, 1941, fig. 1 y 2) (Fig. 7). Sin embargo, lo que caracteriza el vaso en estudio es que dicho detalle se asocia a una forma del cuerpo lisa, que, aunque tampoco es excepcional, pues se conocen ejemplares asirios del Palacio de Sargón II en Nimrud (Schneider-Herrmann, fig. 6) y del Luristán, tanto conservados en museos (Schneider-Herrmann, fig. 12 y 13) como hallados en excavaciones (Haerenick y Overlaet, 1999, 30, fig. 15,8-9), sí resulta mucho menos habitual. Más excepcional es otro cuenco de la Colección Foroughi con el cuerpo liso y ovas en el umbo que ofrece una inscripción aramea fechada hacia el 600 a.C. con nombres acadios (Dupont-Sommer, 1964, 115 s., lám. 37), detalle que evidencia la dificultad que ofrece precisar el origen de estas piezas.
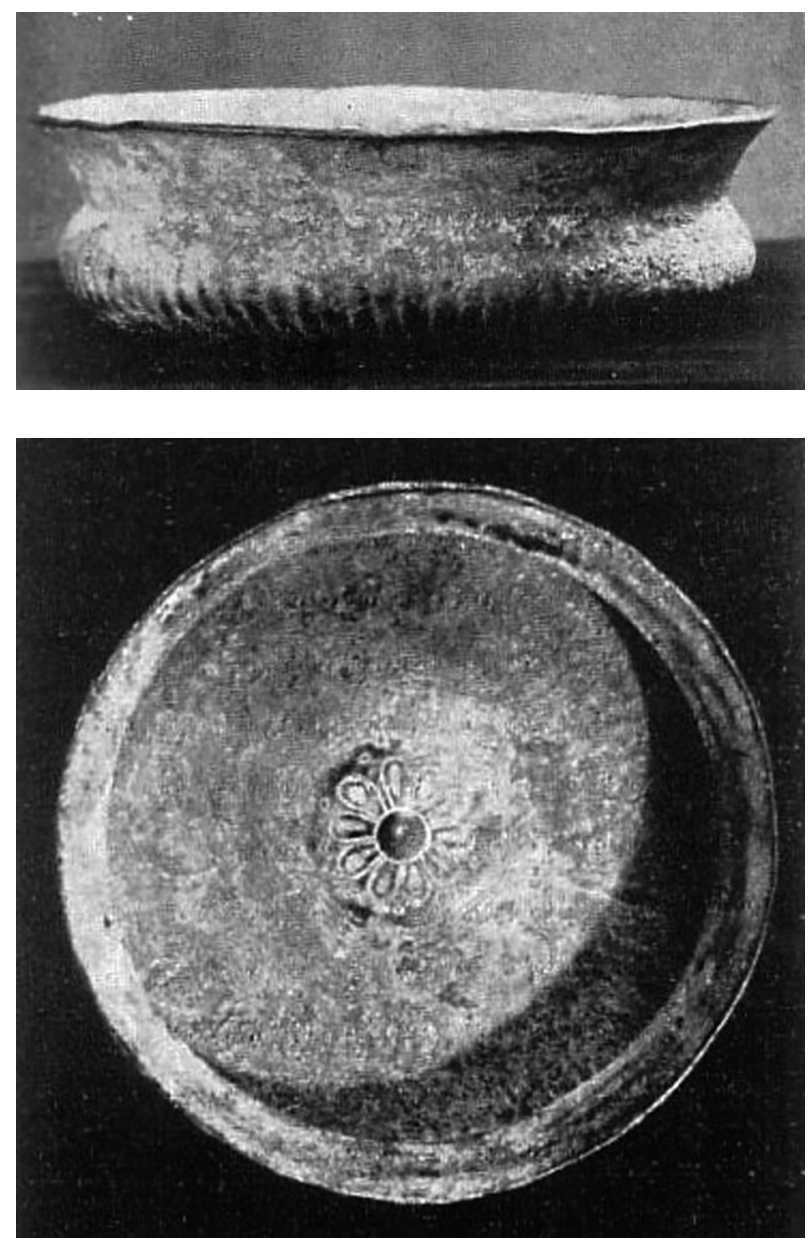

Figura 7: Cuenco del Luristán del Museo de Leiden (según Schneider-Herrmann, 1941).

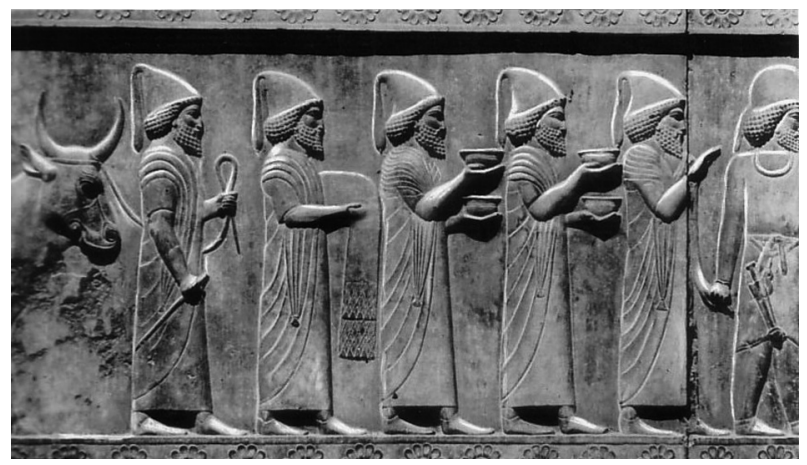

Figura 8: Relieve de Persépolis con las ofrendas de los babilonios a Darío.

Este tipo de phiale, habitualmente decorada con gallones, pasó a ser el vaso característico, por excelencia, del mundo aqueménida (Byvank-Quarles van Ufford, 1978, 59-60, 179-187; 1988, 180-188; 1991, 159-164; Amandry, 1958, 13 ss., 18; Muscarella, 1972, 25-50; Carter, 2001, 163-185; Gunter y Root, 1998: 2-38; etc.), donde en ocasiones adoptan la forma de complejas estructuras florales (Luschey, 1939: fig. 15-21 y 31 ss.; Byvank-Quarles van Ufford, 1983, 179-181), generalmente derivadas de la forma de loto egipcia originaria, y llegan a ofrecer complejas decoraciones (Moorey, 1988, 231-246), como la magnífica phiale de bronce con diez lotos de Dumavizah, en el Luristán (Vanden Berghe, 1966, 96, lám. 124c), conociéndose vasos de este tipo de oro y de plata, en ocasiones con formas más profundas, que en algunos casos llevan los nombre de los soberanos persas, como la phiale de oro con el nombre de Darío (Girshman, 1964, fig. 310) y la copa aúrea con inscripción de Jerjes I (486-465 a.C.) procedentes de Hamadan, la antigua Erbatana (Girshman, fig. 309; Vanden Berghe, 1966, 109, lám. 136c), u otra (Gunter y Root, 1998, 2-38; Gunter y Jett, 1992, 69 s.), con el nombre de Artajerjes (465-425 a.C.).

Su uso debió estar bastante generalizado en todo el Imperio Persa, como indican las repetidas representaciones de este tipo de cuenco en los relieves de los frisos que decoraban la gran escalinata norte del palacio de Darío I en Persépolis, construida hacia el 518 a.C., en la que llevan en sus manos cuencos de esta forma diversas personas de los 23 pueblos del imperio que desfilan ante Darío para presentarle sus tributos (Roaf, 1983, 49 s. fig. 53 y 61); en concreto (Fig. 8), los babilonios (Girshman, 1964, fig. 223; Wilber, 1969, 26; Vanden Berghe, 1966, 31, lám. 34b), lidios (Wilber, 1969: 26; Herzfeld, 1988, lám. 78,b), bactrianos (Wilber, 1969, 27; Vanden Berghe, 1966, 32, lám. 35b), y otros también (Herzfeld, 1988, lám. 78,a), como sirios (Fig. 9) y jonios (Wilber, 1969, 27; Vanden Berghe, 1966, 32, lám. 35a). Además, este tipo de vaso también fue realizado en jaspe gris (Wilber, 1969, 90) y cristal de roca (Damerji, 1998, fig. 24) e imitado en vidrio desde época orientalizante (Fukai, 
1978, 19, fig. 5-7; Young, 1981, 32, fig. 18, lám. 15,AB; Grose, 1989, 80 ss., fig. 48, $n^{\circ} 34$ ) hasta la helenística (Byvanck-Quarles van Ufford, 1970, 129-141) y también desde época geométrica fue imitado en cerámica (Luschey, 1939, fig. 8 a 11), tradición que, en ciento modo, puede considerarse que perduró hasta dar lugar a los llamados vasos «megáricos» (Pagenstecher, 1909; Byvanck-Quarles van Ufford, 1953, 1-21; Kraus, 1951; etc.).

Pero estas formas de cuencos abiertos y carenados también aparecen en el ámbito fenicio orientalizante, a través del cual alcanzaron Etruria e incluso la Península Ibérica, como evidencia el vaso de plata hallado en una tumba de Cástulo (Blanco Freijeiro, 1960), así como la misma Grecia, donde este tipo de formas fueron ampliamente conocidas y utilizadas, pues, desde el mundo aqueménida pasaron al mundo griego (von Bothmer, 1962, 154-166; 1981, 194 s.) y acabaron por generalizarse incluso por el ámbito tracio (ByvankQuarles van Ufford, 1989, 205-219), donde este tipo de phiale aparece en los tesoros de Panagyurishte, a fines del siglo IV, y de Rogozen, formado por más de $20 \mathrm{~kg}$ de plata (Nikolov, 1989, 189-196; ByvanckQuarles van Ufford, 1990, 51-72) y en el área escita del Cáucaso y las estepas (Smirnov, 1934, lám. VIIIXII; Leskov, 2008, 114 ss., $n^{\circ}$ 147) lo mismo que en las montañas del Luristán.

Esta forma metálica tuvo también imitaciones cerámicas, que evidencian su popularidad. En Grecia, ya desde inicios del siglo VI a.C., se utilizaba para las libaciones religiosas un recipiente cerámico derivado de esta forma metálica oriental, la phiale (Luschey, 1939), en algunos casos incluso con la forma caliciforme característica de las piezas aqueménidas, por lo que fueron denominadas Achaemenid Phialai por Strong, forma de la que parece derivar, hacia el 400 a.C., el Kalix Cup (Sparkes, 1970, 121) o forma Lamboglia 41 de las cerámicas áticas y de barniz negro del Mediterráneo Occidental (Lamboglia, 1952, 188).

Se trata de un pequeño vaso de pared cóncava en su parte superior y forma globular en la inferior, desprovisto de asas y de pie, pero con un umbo central realzado característico. Aunque B. Sparkes lo relacionó morfológicamente como una variante del kantharos (Sparkes y Talcott, 1970, 121-122), en realidad, procede de los prototipos metálicos señalados, siendo el kantharos metálico una forma que puede considerarse derivada del prototipo oriental al añadírsele pie y asas.

Estos vasos eran una forma rara en Grecia, bien conocida en usos sacros pero no frecuente, aunque se conocen piezas procedentes de Perachora (Dunbabin, 1951, 61-72, fig. 2) y de las excavaciones de Olinto (Robinson, 1950, 294, láms. 190, 191), donde este vaso, que ofrece gallones en la panza, se denominó Mesomphalic Kantharus y se fechó c. 375/350 a.C., y de Corinto (Williams II, 1972, 159, lám. 25, no 30), datadas en el segundo cuarto del siglo IV a.C. También se conocen phiales de ese tipo en el Ágora de

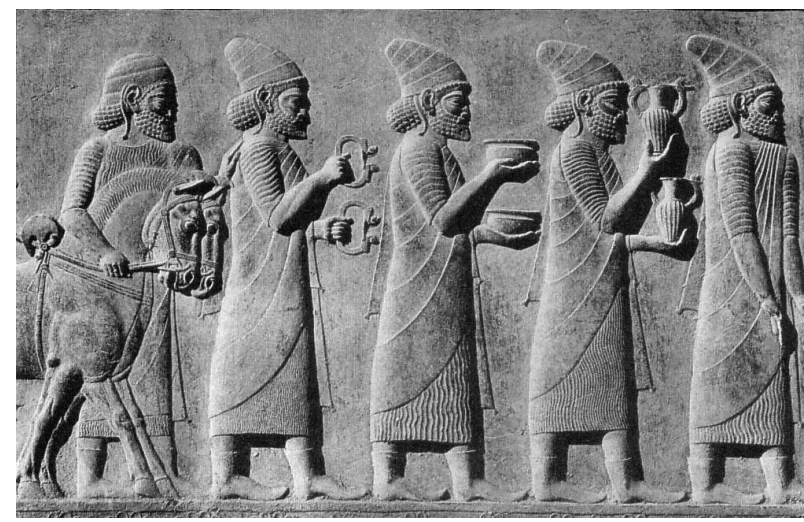

Figura 9: Relieve de Persépolis con las ofrendas de los sirios a Darío.

Atenas, donde se ha podido precisar su evolución. Las formas más antiguas ofrecen la parte superior del vaso baja y abierta, más próxima a los prototipos metálicos aqueménidas, como la hallada en el Depósito G. 13:7 de una casa del Ágora fechada por Sparkes c. 400/350 a.C. (Sparkes, 1970, I, 285; 1970, II, lám. $28, \mathrm{n}^{\circ} 691$ ); por el contrario, las formas más recientes corresponden al denominado Calyx-cup hallado en la Pira de Sacrificio ${ }^{\circ} 8$ del Ágora, fechada ya a fines del siglo IV o inicios del III a.C. (Young, 1951, 124, lám. $52 \mathrm{~b}$ ). En el Mediterráneo Oriental esta forma aparece representada en la procesión citada del friso de la escalera norte de Persépolis, en la que alguno de los participantes lleva un Kalyx-cup en las manos, por lo que se fechan en tiempos de Darío I, hacia el 518 a.C. (vid. supra), y también se ha señalado su perduración en época helenística en Alejandría (Brecia, 1912, lám. LVI, $n^{\circ} 118$ y 124$)$.

En el Mediterráneo Occidental estas imitaciones llegaron en época tardía y fueron todavía más raras, como ya observó N. Lamboglia $(1952,188)$, quien sólo recogió un caso de Ensérune que consideró una forma aislada, a los que cabe añadir algún otro como el publicado por A. García Bellido procedente de la necrópolis de Orán, que consideró suditálica (García Bellido, 1957, lám. 30), aunque J-P. Morel acertadamente la identificó como ática (Morel, 1980, 63, fig. 8), o el procedente del santuario ibérico de La Serreta, en Alcoy (Abad, 1983, 173-197), y otro ejemplar hallado en el pecio de El Sec (Cerdá, 1987, 330-331, $\mathrm{n}^{\circ} 283$, lám. V, 283) entre más de 400 piezas de cerámica de barniz negro, lo que confirma su rareza e, indirectamente, su función ritual como vaso para libación.

La escasez de estas piezas en el Mediterráneo debe relacionarse con un aspecto muy interesante de estas copas, normalmente soslayado por no considerarse de interés o por darse por conocido: su carácter simbólico asociado a su función ritual habitual. En este sentido, conviene tener en cuenta que todo el motivo central, en especial el omphalos, ofrece un preciso significado, probablemente de tipo ctónico, uránico y cosmológico. 
En los ejemplares de bronce, las lengüetas de su parte externa representan la flor de loto, símbolo de vida y de resurrección en todo el Oriente, donde esta flor significaba una alusión a la divinidad y, quizás, a la realeza, como símbolo del renacimiento y de la renovación de la tierra y, en consecuencia, de la fecundidad y la vida, lo que explica su amplia difusión (Barnett, 1974, 18 s.; 1975, lám. 86-87). A este respecto, es interesante que la roseta pasó a ser el motivo decorativo complementario más habitual del arte asirio (Albenda, 2005, lám. $2,5,6,9$, etc.) y todavía más del aqueménida, no sólo en los cuencos de metal, sino también en los bordes de los relieves (Roaf, 1983, lám. 9 y 27), como se puede ver en los de Pasagarda, atribuidos a artistas jonios (Nylander, 1970, 139 ss., fig. 48a y 49b), seguramente recogiendo este significado y el consiguiente carácter apotropaico y benefactor. Pero las lengüetas en estos vasos están dispuestas en torno a un umbo central claramente resaltado por un pequeño saliente, por lo que este detalle ofrece un claro carácter onfálico que confirma que estos cuencos debían tener un profundo simbolismo cosmológico, que explica su aprecio y amplia difusión en la Antigüedad, en distintos ritos de libación y ofrenda.

La pieza de la colección madrileña ofrece la característica específica del umbo central rodeado de ovas semicirculares, que es específica de las piezas del Luristán (Amiet, 1976, 96-98), y que, en principio, permite precisar su procedencia en dicha región. Aunque sea más difícil precisar su cronología, su forma es todavía muy baja y con el borde ya bien diferenciado, por lo que puede considerarse una pieza relativamente antigua en comparación con los vasos aqueménidas, fechable, dentro de las producciones del Luristán, en un momento posiblemente avanzado, hacia el siglo VII a.C. Sin embargo, el umbo rodeado de ovas semicirculares debe interpretarse como una característica ya avanzada dentro de esta serie de vasos, pues es el detalle más próximo a las piezas aqueménidas, lo que llevaría a fechar este ejemplar a partir de finales del siglo VII o inicios del VI a.C., sin que parezca lógico rebasar esta fecha.

\section{EL CUENCO GAYANGOS RAH 982}

El cuenco del Luristán de una colección madrileña se añade a las muy raras antigüedades aqueménidas existentes en España. Junto a él apenas cabe incluir otro vaso que ofrece una forma relacionada y que se conserva en la Real Academia de la Historia, además de un tercer vaso con inscripción recientemente publicado del Museo de Pontevedra (Jaramago, 2005, 303-312). A estos vasos sólo cabe añadir, por su rareza y por ser prácticamente desconocido, un pequeño fragmento de inscripción de sólo $12,5 \mathrm{~cm}$ procedente de Susa, que se conserva en la Real Academia de la Historia (RAH, I.1.1.C-4), donado por D. Adolfo Ribadeneyra a fines del siglo XIX, quien dijo haberlo recogido

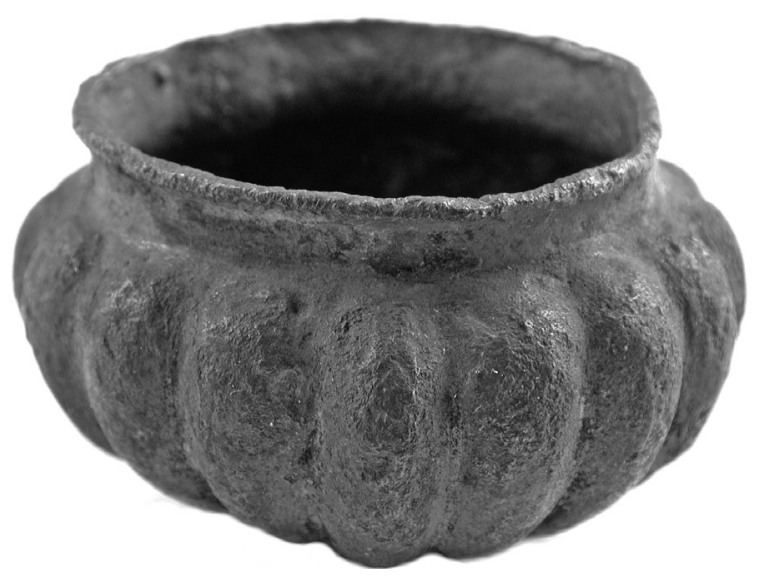

Figura 10: Vaso agallonado de la Colección Pascual de Gayangos de la Real Academia de la Historia.

personalmente en el sepulcro de Dario I en Susa (García y López, 1903, 23, nº 121; Molina, 2003, 474).

El pequeño cuenco de bronce que conserva la Real Academia de la Historia puede considerarse relacionado con estas producciones de vasos orientales. Se trata de un cuenco agallonado que forma parte de la Colección Pascual Gayangos y Arce (1809-1897), famoso erudito, historiador, arabista y bibliógrafo (Marqués de Siete Iglesias, 1978, 325-329, $\mathrm{n}^{\circ}$ 125; Manzanares, 1971, 81 ss.; Vallvé, 1997, 459-488), cuya pequeña pero interesante colección de antigüedades fue donada tras su muerte a la Real Academia de la Historia en 1898 por sus hijos y herederos, Emilia de Gayangos de Riaño y José de Gayangos (Fita y Rodríguez Villa, 1898,93 ), por lo que se conserva en la actualidad en esta institución (García y López, 1903, nº 982; Almagro-Gorbea, 2010).

La colección Pascual de Gayangos llegó sin inventario de las piezas, por lo que se carece de noticia alguna sobre la procedencia originara de este interesante vaso y de las restantes piezas que la componen, como tampoco se sabe cómo llegó a poder del Sr. Gayangos, quien pudo recogerlo en alguno de sus viajes por Oriente o por cualquiera de las capitales europeas en una de sus visitas, pues la variada procedencia de las antigüedades de su colección impide conocer su origen. Por ello, la única alternativa es la información que ofrece el estudio tipológico de la pieza.

Se trata de un pequeño cuenco de bronce que ofrece una forma característica constituida por un cuerpo inferior agallonado y un pequeño borde ligeramente exvasado. Pudo estar realizado a torno, como indicaría un ligero rehundido en su base externa, pero en la actualidad está algo deformado y no se puede asegurar dicho detalle técnico. Sus paredes son relativamente gruesas, de c. $2 \mathrm{~mm}$, aunque este grosor no es regular a causa de los gallones (Fig. 10).

Presenta un perfil ligeramente en $\mathrm{S}$, con un cuello cóncavo, relativamente bajo, de $6 \mathrm{~mm}$. de alto, seguido de un cuerpo agallonado que ofrece su mayor 
curvatura en la parte superior. Este cuerpo inferior es bastante profundo y su forma agallonada es la habitual en este tipo de cuencos. Ofrece 16 gallones bien marcados en el exterior, pero apenas en el interior, lo que parece indicar que la pieza ha sido realizada a la cera perdida. Los gallones no son simétricos, sino que vistos desde la base unos resultan ligeramente más alargados que otros, lo que da a la pieza cierto aspecto de tosquedad. Además, en la base presenta una pequeña oquedad circular de $4 \mathrm{~mm}$ de diámetro por 1,2 $\mathrm{mm}$ de profundidad, a modo de umbo, pero que no se aprecia por el interior, oquedad que queda en posición ligeramente asimétrica.

El estado de conservación puede considerarse satisfactorio pues no ofrece roturas, aunque el borde aparece deformado y queda cubierto por una capa de cuprita rojiza algo irregular, que en el interior alterna con restos de sales y óxidos de cobre.

Dimensiones: Diámetro máximo: 50/55 mm. Diámetro de la boca: $42 \mathrm{~mm}$. Altura máxima: $36 \mathrm{~mm}$. Peso: 90.50 gr. Capacidad de la panza: $30 \mathrm{~cm}^{3}$. Capacidad hasta el borde: $38 \mathrm{~cm}^{3}$.

Análisis metalográfico: $\mathrm{Cu}$ 80.9\%; Sn 5,53\%; Pb $13.2 \%$; Fe 0.13\%; Ni nd; Zn nd; As nd; Ag 0.010\%; Sb $0.185 \%$; Bi nd ${ }^{2}$.

La forma de este pequeño vaso de la Real Academia de la Historia debe considerarse relacionada con los numerosos vasos agallonados que se documentan en Oriente desde inicios del I milenio a.C. hasta épocas avanzadas del Imperio persa. Sin embargo, el pequeño tamaño de la pieza y su relativa profundidad lo apartan de las phiales y cuencos semejantes, por lo que, a falta de toda referencia sobre su procedencia, sólo puede compararse por su forma a algún paralelo algo alejado y ciertamente más tardío (Moorey, 1981, $133, n^{\circ} 655$ ), como confirmaría su análisis metalográfico, lo que lleva a suponer que esta pieza, de fabricación relativamente tosca, pudiera incluirse dentro de la tradición de estos vasos pero ya hacia mediados del I milenio a.C., sin excluir, dada la citada falta de paralelos conocidos, la segunda mitad del mismo, pudiendo

\footnotetext{
2. Análisis efectuado con el espectómetro de fluorescencia de rayos X (energías dispersivas) Metorex XMET920 del Laboratorio del Museo Arqueológico Nacional por el Dr. Salvador Rovira, a quien agradecemos esta nueva colaboración amistosa.

Según su informe metalográfico (14.5.2009), este cuenco agallonado presenta una aleación ternaria $\mathrm{Cu}-\mathrm{Sn}-\mathrm{Pb}$ apta para el modeo de piezas con poco espesor de metal, pero que no se presta bien al laminado, porque el mucho plomo fragiliza el material. Esta pieza presenta una tecnología claramente diferenciada de la que ofrece el cuenco del Luristán (vid. supra, n. 3), que es de una tradición más antigua que se remonta a la Edad del Bronce. En cambio, las vasijas como ésta, de pared fina obtenidas a molde, son más tardías, con una cronología para las más tempranas que no remonta más allá de los siglos VI-V a.C. en materiales griegos y etruscos. Una tecnología no sustituyó a la otra, pues ambas han pervivido hasta nuestros días.
}

tratarse de un producto del Luristán o de cualquier otro centro metalúrgico de Oriente.

\author{
Dr. Martín Almagro Gorbea \\ Real Academia de la Historia \\ c/ León, 21 \\ 28014 Madrid \\ anticuario@rah.es \\ Dr. Joaquín López Pascual \\ Secretario General \\ Colegio de estudios Financieros \\ c/ Serrano Anguita, 9 \\ 28004 Madrid \\ joaquinlopez@cunef.edu
}

\section{BIBLIOGRAFÍA}

ABAD, L., 1983: «Un conjunto de materiales de La Serreta de Alcoy», Lucentum, 2, 173-197.

ALBENDA, P., 2005: Ornamental Wall Painting in the Arte of the Assyrian Empire, Leiden.

ALMAGRO-GORBEA, M., 2003: «Una pátera fenicia de Nubia y el comercio fenicio en los confines del Mundo Antiguo», Complutum, 14, 7-32.

ALMAGRO-GORBEA, M., 2010: «La colección de antigüedades de Pascual de Gayangos», en G. ANES y ÁLVAREZ DE CASTRILLÓN (Coord.), Pascual de Gayangos en el bicentenario de su nacimiento, Madrid, 139-144.

AMANDRY, P., 1958: «Orfevrerie achemenide», Antike Kunst, 1, 9-23.

AMIET, P., 1976: Les antiquités du Luristan. Collection David-Weill, Paris.

ANDRAE, W., 1943: Ausgrabungen in Sendschirli, V, Berlin.

AYOUB, A., 1982: Die Keramik in Mesopotamien und in den Nachbargebieten. Von der Ur III-Zeit bis zum Ende der kassitischen Periode, München.

BARNETT, R.D., 1975: A Catalogue of the Nimrud Ivories, London, $2^{\mathrm{a}}$ ed.

BARNETT, R.D., 1974: «The Nimrud Bowls in the British Museum», Rivista di Studi Fenici, 2, 11-33.

BLANCO FREIJEIRO, A., 1960: «Orientalia II», Archivo Español de Arqueología, 33, 3-43.

BOTHMER, D. F. von, 1962: «A Gold Libation Bowl», Bulletin of the Metropolitan Museum of Art, December 1962, 154-166.

BOTHMER, D. F. von, 1981: «Les trésors d'orfèvrerie de la Grèce orientale au Metropolitan Museum de New York», Comptes Rendues de l'Académie des Inscriptions et Belles-Lettres, 125:2, 194-207.

BYVANK-QUARLES VAN UFFORD, L., 1953: «Les bols mégarines», Bulletin Antieke Beschaving, 28, 1-21.

BYVANK-QUARLES VAN UFFORD, L., 1970: «Les bols hellénistiques en verre doré», Bulletin Antieke Beschaving, 45, 129-141.

BYVANK-QUARLES VAN UFFORD, L., 1978: «Une coupe ionienne de la Basse Epoque achéménide», Oudheidkundige Mededelingen Leiden, 59-60, 179-187. 
BYVANK-QUARLES VAN UFFORD, L., 1983: «Une coupe achéménide d'argent du Musée des Antiquités de Leyden», Bulletin Antieke Beschaving, 58, 179-181.

BYVANK-QUARLES VAN UFFORD, L., 1988: «L'art préclassique et le style archaïsant», Bulletin Antieke Beschaving, 63, 180-188.

BYVANK-QUARLES VAN UFFORD, L., 1989: «À propos de l'orfevrèrie thrace», Bulletin Antieke Beschaving, 64, 205-219.

BYVANK-QUARLES VAN UFFORD, L., 1990: «À propos du trésor de Rogozen», Bulletin Antieke Beschaving, 65, 51-72.

BYVANK-QUARLES VAN UFFORD, L., 1991: "'Achämenidischer Becher' ou 'bol ionien à panse arrondie'?», Bulletin Antieke Beschaving, 66, 159-164.

CALMEYER, P., 1964: Altiranische Bronzen der Sammlung Bröckelschen, Berlin.

Carter, m., 2001: «Preliminary notes on seven Achaemenid silver objects in the Miho Museum», Studia iranica, 30, $2,163-185$

CERDÁ, D., 1987: «La cerámica ática de barniz negro», en A. ARRIBAS, G. TRÍAS, D. CERDÁ y J. DE HOZ, El barco de El Sec (Costa de Calviá, Mallorca), Mallorca, 197-385.

DAMERJI, M.S.B., 1998: «Gräber assyrischer Königinnen aus Nimrud», Jahrbuch des Römisch-Germanischen Zentralmuseums, 45, 597-659.

DUNBABIN, T. J., 1951: "The Oracle of Hera Akraia at Perachora», Annual of the British School of Athens, 46, 61-72.

DUPONT-SOMMER, A., 1964: «Trois inscriptions araméennes inédites sur des bronzes du Luristan», Iranica Antiqua, 4, 108-118.

FITA, F. y RODRÍGUEZ VILLA, A., 1898: «Noticias», Boletín de la Real Academia de la Historia, 32,1, 93.

FUKAI, S., 1977: Persian Glass, New York-Kyoto.

GARCÍA BELLIDO, A., 1957: «La necrópolis de Orán», Archivo Español de Arqueología, 30, 90-106.

GARCÍA Y LÓPEZ, J. C., 1903: «Inventario de las Antigüedades y Objetos de Arte que posee la Real Academia de la Historia», $B R A H, 42,1903,311-316,321-368,484-$ 505 y $B R A H, 43,1903,257-322$, Madrid.

GIRSHMAN, R., 1964: Iran. Protoiranier, Meder, Achämeniden, München.

GODARD, A., 1931: Les bronzes du Luristan, Paris.

GROSE, D. F., 1989: The Toledo Museum of Art. Early Ancient Glass... 1600 B.C. to A.D. 50, New York.

GUNTER, A. y ROOT, M., 1998: «Replicating, inscribing, giving: Ernst Herzfeld and Artaxerxes silver phiale in the Freer Gallery of Art», Ars Orientalis, 28, 3-40.

GUNTER, A.C. y JETT, P., 1992: Ancient Iranian Metalwork in the M. Sackler Gallery and the Freer Gallery of Art, Washington.

HAERENICK E. y OVERLAET, B., 1999: Djub-i Gauhar und Gul Khanan Murdah Iron Age III graveyards in the Aivan plain (Luristan Excavation Documents III), Brussels.
HAERENICK, E. y OVERLAET, B., 1998: Chamahzi Mumah. An Iron Age III Graveyard (Luristan Excavation Documents II), Brussels.

HERZFELD, E. E., 1988: Iran in the Ancient East, New York.

JARAMAGO, M., 2005: «El vaso aqueménida del Museo de Pontevedra: un análisis epigráfico», El Museo de Pontevedra, 59, 303-312.

KRAUS, Th., 1951: Megarische Becher im Römisch-Germanischen Zentralmuseum zu Mainz, Mainz.

LAMBOGLIA, N., 1952: Per una classificazione preliminare della ceramica campana, Bordighera.

LESKOV, A. M., 2008: The Maikop Treasure, Philadelphia.

LUSCHAN, F. Von, 1943: Die kleine Funde von Sendschirli (Ausgrabungen in Sendschirli, V), Berlin.

LUSCHEY, H., 1939: Die Phiale, Bleicherode-am-Harz.

MALLOWAN, M. E. L., 1966: Nimrud and its Remains, I, London.

MANZANARES, M., 1971: Arabistas españoles del siglo $X I X$, Madrid.

MOLINA, M., 2003: «Inscripciones cuneiformes», en M. ALMAGRO-GORBEA, Catálogo de la Real Academia de la Historia, I.1.1, Epigrafia Prerromana, Madrid, 471-474.

MOOREY, P. R. S., 1981: Ancient Bronzes, Ceramics and Seals. The Nasli M. Heeramaneck Collection of Near Eastern, Central Asiatic and European Art, 133, $\mathrm{n}^{\circ} 655$, Los Angeles.

MOOREY, P. R. S., 1988: «The technique of gold-figure decoration on achaemenid silver vessels and its antecedents»), Mélanges P. Amiet (Iranica Antiqua 23), 231-246.

MOOREY, P. R. S., 1974: Ancient Persian Bronzen in the Adam Collection, London.

MOREL, J.-P., 1980: Céramique campanienne. Les formes, Rome.

MUSCARELLA, O. W., 1972: «A Bronze Vase from Iran and Its Greek Connections», Metropolitan Museum Journal, 5, 25-50.

MUSCARELLA, O. W., 1988: «The Background to the Luristan Bronzes», en J. CURTIS (ed.), Bronzeworking Centres of Western Asia c. 1000-539 B.C., 33 s, LondonNew York.

NIGRO, L., 2002: «The Middle Bronze Age Pottery of Nothern Inner Syria», en M. AL-MAQDISSI, V. MATOÏAN y CHR. NICOLLE (eds.), Céramique de l'Âge du Bronze en Syrie, I. La Syrie du Sud et la vallée de l'Oronte, 118, no 35-43, Beyrouth.

NIKOLOV, B., 1989: «Le trésor thrace de Rogozène, dép. de Vratsa, Bulgarie», Thraco-Dacica, 10,1-2, 189-196.

NYLANDER, C., 1970: Ionians and Pasagardae, Uppsala.

PAGENSTECHER, R., 1909: Die calenische Reliefkeramik, Berlin.

ROAF, M., 1983: Sculptures and Scuptors at Persepolis (Iran 21), London.

ROBINSON, D. M., 1950: Excavations at Olynthus, XIII. Vases found in 1934 and 1938. London. 
SCHNEIDER-HERRMANN, G., 1941: «Een bronzen oostersche schaal uit particulier vestí in Leiden», Bulletin Antieke Beschaving, 16,2, 17-22.

SIETE IGLESIAS, Marqués de, 1978: «Real Academia de la Historia. Catálogo de sus individuos. Noticias sacadas de su archivo», Boletín de la Real Academia de la Historia, 125, 309-352.

SMIRNOV, J. I., 1934: Der Schatz von Achalgori, Tiflis.

SPARKES, B. y TALCOTT, L., 1970: Black and Plain Pottery of the $6^{\text {th }}, 5^{\text {th }}$ and $4^{\text {th }}$ Centuries B.C. (The Athenian Agora XII), Princeton.

VALLVÉ, J., 1997: «Pascual de Gayangos (1809-1897). A propósito del centenario de su muerte», $B R A H$, CXCI, 459-488.

VANDEN BERGHE, L., 1952: Archéologie de l'Iran Ancien, Leiden.
VANDEN BERGHE, L., 1967: «La nécropole de War Kabud ou le déclin d'une civilisation du bronze», Archeologia, 18, 48-61.

VANDEN BERGHE, L., 1968: Het archeologisch onderzoek naar de Bronzecultuur van Luristan. Opgravingen in Pusht-i Kush I (1965 en 1966), Brussel.

VANDEN BERGHE, L., 1979: «Les bronzes du Luristan de l'Âge du Fer III. Resultats des fouilles aun Pushti Kuh», Akten des VII. Internationale Kongresses für Iranische Kunst und Archäologie, München-1976 (Archäologische Mitteillungen aus Iran, Ergänzungsband 6), Berlin, 138-150.

WAELE, E. De, 1982: Bronzes du Luristan et d'Amlash. Ancienne Collection Godard, Louvain-la-Neuve.

WILBER, D. N., 1969: Persepolis. The Archaeology of Parsa, Seat of the Persian Kings, London.

YOUNG, R. S., 1981: The Gordion Excavations Final Reports I. Three Great Early Tumuli, Pennsylvania. 